ORIGINAL ARTICLE

\title{
Effect of Early Rehabilitation in the Intensive Care Unit by a Dedicated Therapist Using a Rehabilitation Protocol: A Single-center Retrospective Study
}

\author{
Kohji Iwai, PT, PhD a Tomoyuki Hisano, PT a Ryo Komada, PT a Tamami Miyai, PT a Kazutaka Sakai, PT a \\ Mayu Torimoto, $\mathrm{PT}^{\mathrm{a}}$ and Yasuyuki Tsujita, MD, $\mathrm{PhD}^{\mathrm{b}}$
}

\begin{abstract}
Objectives: This study investigated retrospectively the effect of early rehabilitation in the intensive care unit (ICU) by a dedicated therapist using a rehabilitation protocol. Methods: The subjects comprised patients admitted to our emergency ICU. A dedicated therapist and a rehabilitation protocol were instigated in April 2018. We enrolled 330 patients in phase I (April 2016-March 2018) and 383 patients in phase II (April 2018-March 2020). Patients in the ICU for only one night and pediatric patients were excluded. The following data were accessed from medical records: sex, height, age, Sequential Organ Failure Assessment, rehabilitation intervention, ventilation at admission, duration of mechanical ventilation, extubation, reintubation, tracheotomy, length of ICU stay, length of hospital stay, and outcome. The effectiveness of rehabilitation was assessed using the time from ICU admission to the first rehabilitation session, first sitting exercise, and first standing exercise. Clinical outcomes were analyzed separately for subjects discharged to home or transferred to another hospital. Results: The percentage of subjects undergoing rehabilitation intervention increased significantly from $23.4 \%$ to $56.7 \%(\mathrm{P}<0.001)$ in phase II. Moreover, reintubation ( $\mathrm{P}=0.045)$; the length of ICU stay $(\mathrm{P}=0.022)$; and the time from ICU admission to the first rehabilitation session $(\mathrm{P}<0.001)$, the first sitting exercise $(\mathrm{P}=0.001)$, and the first standing exercise $(\mathrm{P}=0.047)$ significantly decreased in phase II. Furthermore, the duration of mechanical ventilation $(\mathrm{P}=0.007)$ and the length of ICU stay $(\mathrm{P}=0.036)$ were significantly reduced in the transfer group. Conclusions: Although the effectiveness of early intervention was suggested, prospective multicenter studies are required to confirm this finding.
\end{abstract}

Key Words: dedicated therapist; early rehabilitation; intensive care unit; rehabilitation protocol; retrospective study

\section{INTRODUCTION}

Early mobilization is a core program of early rehabilitation and has the potential to reduce the occurrence of ventilatorassociated pneumonia, ${ }^{1)}$ decrease the duration of mechanical ventilation, ${ }^{2,3)}$ and improve muscle function. ${ }^{4}$ Furthermore, with the growing awareness of intensive care unit-acquired weakness (ICU-AW), ${ }^{5)}$ early rehabilitation during intensive care has become increasingly common in recent years. In Europe, $75 \%$ of ICUs had at least one physical therapist (PT) working exclusively in the ICU.6) However, currently, only $16 \%$ of healthcare facilities in Japan have rehabilitation protocols and only $18 \%$ have dedicated therapists in the ICU.?) Against this background, to promote early rehabilitation in the ICU, a dedicated therapist was assigned to the ICU in our hospital from April 2018, and proactive early rehabilitation was started in compliance with the rehabilitation protocol. Matsuki et al. ${ }^{8)}$ reported that the effects of having a PT exclusively in the ICU included improvements in muscle strength and the ability to perform activities of daily living, as well

Received: April 8, 2021, Accepted: July 14, 2021, Published online: August 4, 2021

${ }^{a}$ Division of Physical Therapy, Rehabilitation Units, Shiga University of Medical Science, Otsu, Japan

${ }^{\mathrm{b}}$ Department of Critical and Intensive Care Medicine, Shiga University of Medical Science, Otsu, Japan

Correspondence: Kohji Iwai, PT, PhD, Seta Tsukinowa-cho, Otsu, Shiga, Japan, E-mail: kojin@belle.shiga-med.ac.jp

Copyright (C) 2021 The Japanese Association of Rehabilitation Medicine

This is an open-access article distributed under the terms of the Creative Commons Attribution Non-Commercial No

Derivatives (CC BY-NC-ND) 4.0 License. http://creativecommons.org/licenses/by-nc-nd/4.0/ 


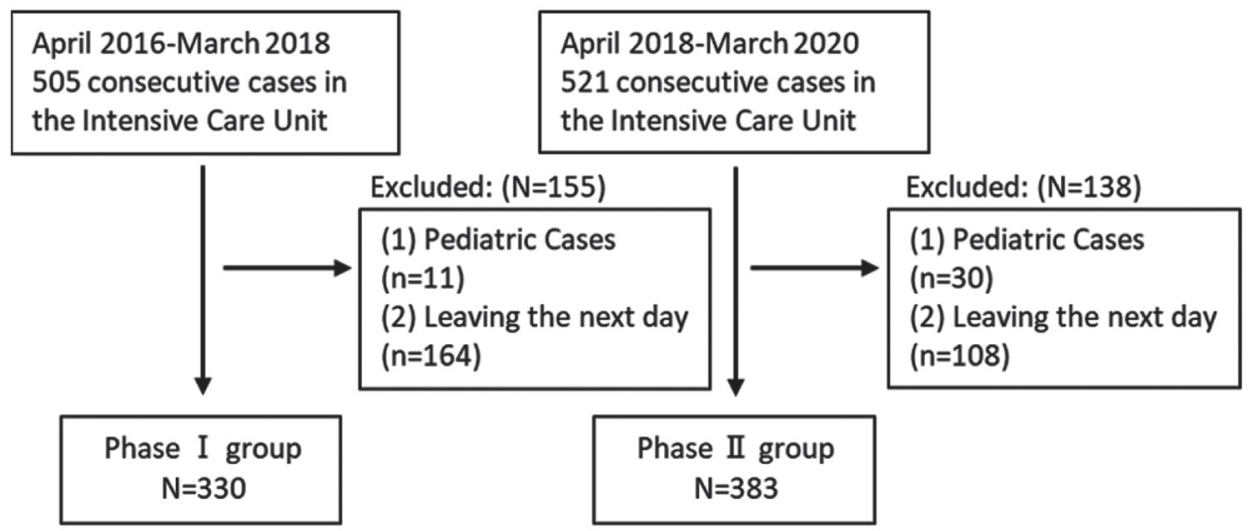

Fig. 1. Flowchart showing patient selection.

as reductions in the length of ICU stay and hospitalization. However, in that study there were many exclusion criteria and the small number of cases may have resulted in bias.

The purpose of the current study was to retrospectively investigate the clinical outcomes of patients admitted to the ICU in the 2 years before and in the 2 years after the initiation of early rehabilitation intervention in the ICU by a dedicated therapist and to validate the effectiveness of the intervention.

\section{METHODS}

Patient information including sex, height, age, and the Sequential Organ Failure Assessment (SOFA) score were recorded at ICU admission. Furthermore, the percentage of patients undergoing rehabilitation intervention; the percentage of patients on a ventilator at admission; the duration of mechanical ventilation; and the percentages of patients undergoing extubation, reintubation, and tracheotomy were extracted from medical records. In our hospital, the main indications for tracheotomy were prolonged mechanical ventilation of more than 2 weeks, patients who were expected to require long-term ventilator management, and after re-intubation. Moreover, hemodynamic instability (tachycardia, arrhythmia, and elevation of blood pressure), increased respiratory rate, a decrease in partial pressure of arterial oxygen, and an increase in partial pressure of arterial carbon dioxide were considered to be indicators for reintubation. ${ }^{9)}$ Furthermore, the length of ICU stay, the length of hospital stay, and the outcome at discharge (discharge to home, transfer, or death) were assessed using medical records. The indices of progress of rehabilitation were the number of days from ICU admission to the day when the patient first underwent rehabilitation, when the patient first sat, and when the patient first stood. When sitting and standing exercises were performed, the therapist provided assistance beside the patient as part of risk management, but the amount of assistance provided was adjusted according to the patient's stability. In the group that underwent rehabilitation intervention, the aspects of the rehabilitation program that were performed (contracture prevention, sitting and standing exercises, electrical muscle stimulation, and cycle ergometer exercise) were investigated. We compared the clinical outcomes of those discharged and those transferred. The assessments included the SOFA score, the number of patients on ventilators at admission, the percentage of extubations, the duration of mechanical ventilation, the length of ICU stay, and the length of hospital stay.

The study protocol conformed to the ethical guidelines of the 1975 Declaration of Helsinki, as reflected in a priori approval by the Clinical Research Ethical Committee of Shiga University of Medical Science Hospital (approval number: 2020-050). Informed consent was obtained on an opt-out basis.

\section{SUBJECTS}

This was a retrospective study of patients admitted to our emergency ICU between April 2016 and March 2020. Patients who underwent cardiovascular surgery and were under coronary care unit management were excluded. During the period from April 2016 to March 2018, no dedicated therapist was present in the ICU; this period is termed phase I and constitutes the control. During the period from April 2018 to March 2020, a dedicated therapist was allocated to the ICU, and this period is termed phase II. We excluded patients who spent only one night in the ICU and pediatric patients under 18 years old. In total, 330 phase-I patients and 383 phase-II 


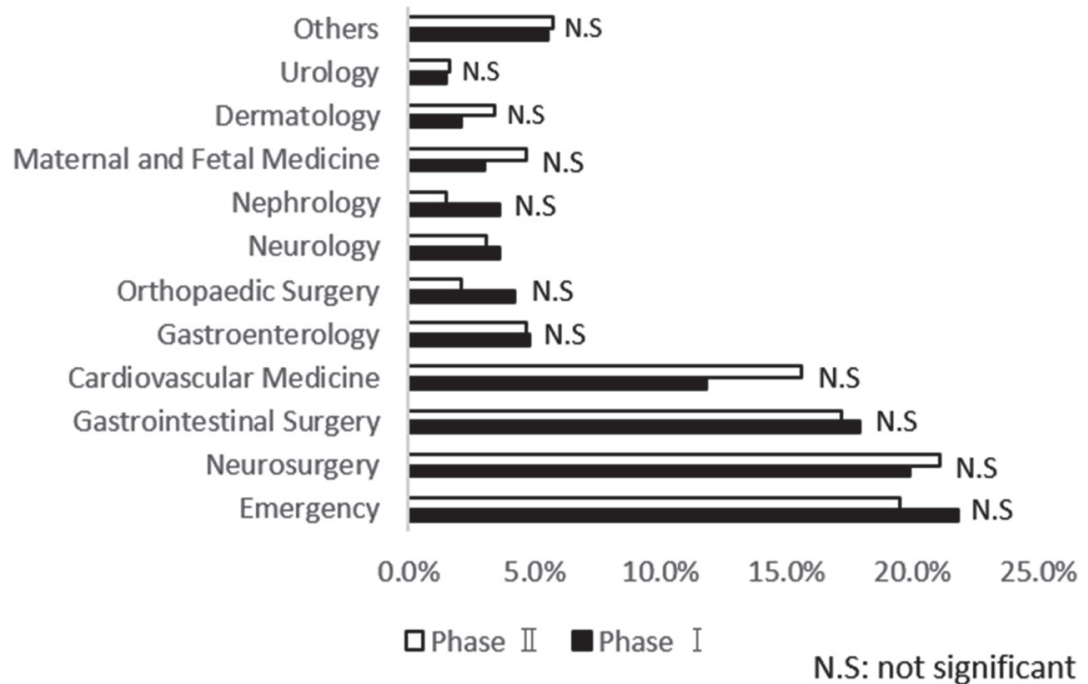

Fig. 2. Proportion of ICU patients in each medical field.

patients were included in this study (Fig. 1). The percentages of patients under the management of the various medical departments are shown in Fig. 2. Sedation and analgesia in mechanically ventilated patients were managed according to the guidelines proposed by the Japan Society of Respiratory Care Medicine. In phase I, early rehabilitation was provided by a PT only in cases where the ICU doctor determined the need for rehabilitation. Other patients were supported by doctors and nurses who shared information and judged the condition of patients. In contrast, in phase II, doctors, nurses, and the dedicated therapist shared patient information, set goals in accordance with the rehabilitation protocol, and implemented early rehabilitation as soon as possible. Early rehabilitation sessions were carried out once a day for 20-60 min per session. The rehabilitation program included contracture prevention, sitting exercises, standing exercises, electrical muscle stimulation, and cycle ergometer exercises; the selection of exercises to be performed was based on the patient's condition. Figure 3 shows the procedure for determining the protocol level and details of the interventions. In addition to rehabilitation, postural drainage was changed every $2 \mathrm{~h}$ by bedside nurses during both phase I and phase II. During the study period, one physical therapist and the ICU doctor were consistently responsible for the exercise rehabilitation regimens of all enrolled patients.

\section{STATISTICS ANALYSIS}

Statistical analyses were performed using IBM SPSS Statistics 22 (IBM, New York, NY, US). Results were con- sidered statistically significant if the two-tailed $\mathrm{P}$ value was less than 0.05. Data are shown as the mean $\pm \mathrm{SD}$, except for non-normally distributed variables, which are shown as the median. For continuous variables, Student's $t$-test or the Mann-Whitney $U$ test was used depending on the validity of the normality assumption. The chi-squared test was used to assess categorical variables. The outcomes at discharge and the prognosis underwent chi-squared tests after crosstabulation.

\section{RESULTS}

A comparison of the basic information of the control and intervention groups is shown in Table 1. The sex ratio was significantly different $(\mathrm{P}=0.005)$, but the SOFA score was not significantly different between the two groups. Furthermore, the proportion of patients undergoing rehabilitation intervention in phase II increased significantly from $23.4 \%$ to $56.7 \%$ $(\mathrm{P}<0.001)$, and reintubation $(\mathrm{P}=0.045)$ and the length of ICU stay $(\mathrm{P}=0.022)$ significantly decreased. The number of days between ICU admission and the day when the patient first underwent rehabilitation $(\mathrm{P}<0.001)$ was significantly reduced in phase II. There were no significant differences in the duration of mechanical ventilation, the percentage of extubations and tracheotomies, the length of hospital stay, discharge outcomes, or mortality. There was no significant difference in the breakdown of the medical departments responsible for the two groups. Comparison of the indices of progress of rehabilitation revealed that the percentage of patients who underwent rehabilitation who achieved sitting 


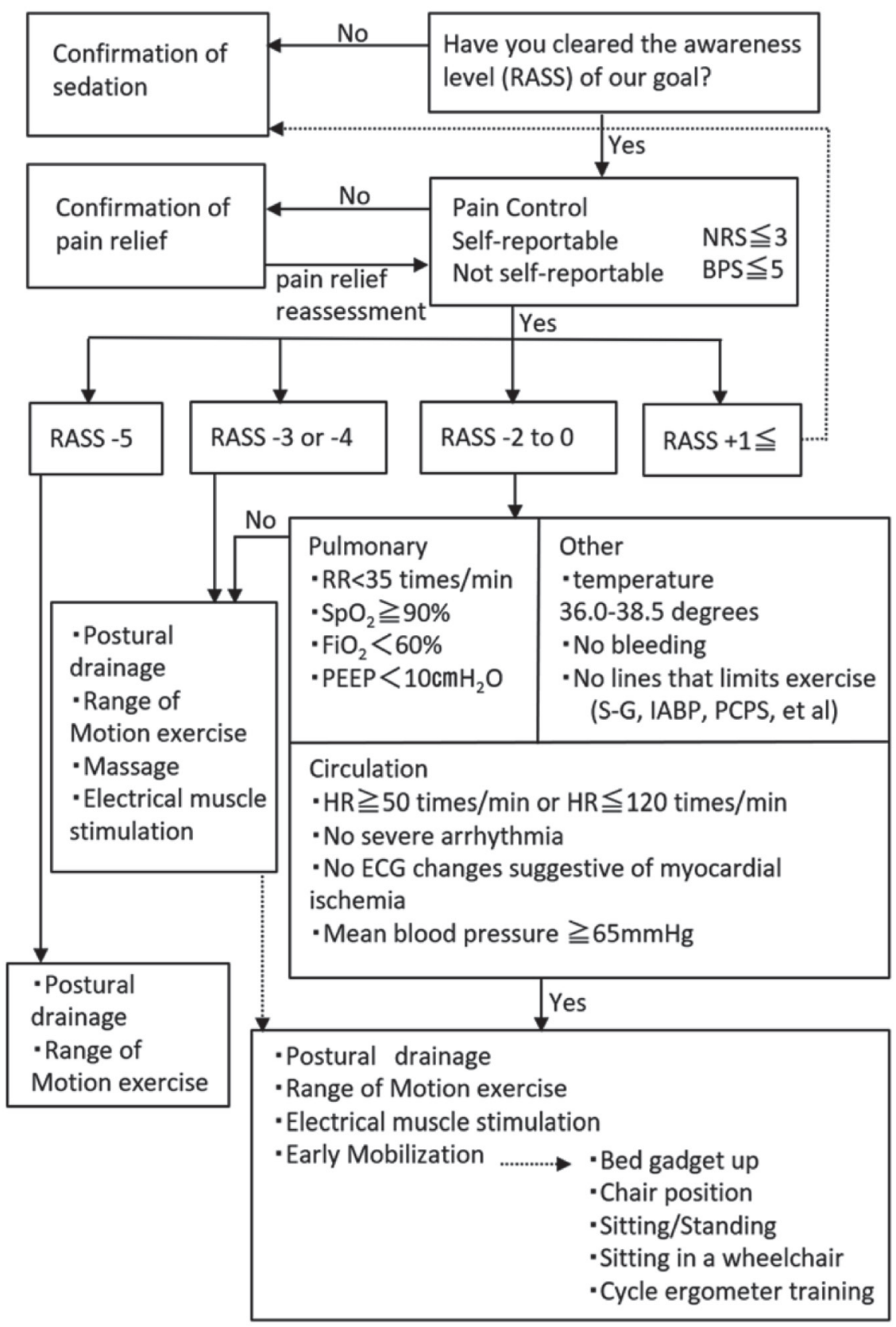

Fig. 3. Rehabilitation protocol for patients in the intensive care unit in our hospital. RASS, Richmond Agitation and Sedation Scale; NRS, numerical rating scale; BPS, behavioral pain scale; RR, respiration rate; PEEP, positive end-expiratory pressure; S-G, Swan-Ganz catheter; IABP, intra-aortic balloon pump; PCPS, percutaneous cardiopulmonary support; HR, heart rate; ECG, electrocardiogram.

on the edge of the bed was significantly higher in phase I, but the day of first sitting $(\mathrm{P}=0.001)$ and the day of first standing $(\mathrm{P}=0.047)$ were significantly reduced in phase II. The percentage of subjects undergoing electrical muscle stimulation $(\mathrm{P}=0.001)$ and cycle ergometer training $(\mathrm{P}=0.044)$ increased significantly in phase II (Table 2). The separate comparison of clinical outcomes in patients discharged to home or transferred to another hospital revealed that the duration of mechanical ventilation $(\mathrm{P}=0.007)$ and the length of ICU stay $(\mathrm{P}=0.036)$ were both significantly reduced in the transfer group. In contrast, there were no significant differences in these parameters in the discharge group (Table 3 ). 
Table 1. Baseline characteristics of phase-I and phase-II ICU patients

\begin{tabular}{|c|c|c|c|}
\hline & $\begin{array}{c}\text { Phase I } \\
2016-2017 \\
(\mathrm{n}=330)\end{array}$ & $\begin{array}{c}\text { Phase II } \\
2018-2019 \\
(\mathrm{n}=383)\end{array}$ & $\mathrm{P}$ value \\
\hline Sex (male, \%) & $232(70.3)$ & $229(59.8)$ & 0.005 \\
\hline Height (cm) & $160.9 \pm 11.6$ & $160.0 \pm 12.2$ & 0.210 \\
\hline Age (year) & $62.4 \pm 18.8$ & $63.0 \pm 19.4$ & 0.582 \\
\hline SOFA score & $7.7 \pm 3.4$ & $7.8 \pm 3.4$ & 0.44 \\
\hline Rehabilitation intervention (n, \%) & $77(23.4)$ & $217(56.7)$ & $<0.001$ \\
\hline Patients on ventilator (n, \%) & $261(79.1)$ & $298(77.8)$ & 0.726 \\
\hline Duration of ventilation (days) & $4.0(2.0-8.0)$ & $3.0(1.0-6.0)$ & 0.436 \\
\hline Extubation (n, \%) & $181(69.3)$ & $192(64.4)$ & 0.218 \\
\hline Reintubation (n, \%) & $16(6.1)$ & $8(2.7)$ & 0.045 \\
\hline Tracheotomy (n, \%) & $57(21.8)$ & $85(26.8)$ & 0.07 \\
\hline Length of ICU stay (days) & $6.0(3.0-12.0)$ & $4.0(3.0-8.0)$ & 0.022 \\
\hline Length of hospital stay (days) & $33.0(20.0-57.0)$ & $36.0(14.0-77.0)$ & 0.616 \\
\hline Discharge to home (n, \%) & $150(45.5)$ & $169(44.1)$ & \\
\hline Transfers (n, \%) & $113(34.2)$ & $134(35.0)$ & 0.922 \\
\hline Death (n, \%) & $67(20.3)$ & 80 (20.9) & \\
\hline
\end{tabular}

ICU; intensive care unit, SOFA; Sequential Organ Failure Assessment.

Table 2. Comparison of rehabilitation program applied in phase II with a dedicated ICU physical therapist

\begin{tabular}{lccc}
\hline & $\begin{array}{c}\text { Phase I } \\
2016-2017 \\
(\mathrm{n}=77)\end{array}$ & $\begin{array}{c}\text { Phase II } \\
2018-2019 \\
(\mathrm{n}=217)\end{array}$ & $\begin{array}{c}\text { P value } \\
\text { SOFA score }\end{array}$ \\
Days to first rehabilitation (days) & $9.3 \pm 3.3$ & $8.6 \pm 3.1$ & 0.067 \\
Prevention of contracture (n, \%) & $4.0(2.5-8.0)$ & $1.0(1.0-1.0)$ & $<0.001$ \\
Sitting on the edge of the bed (n, \%) & $75(97.4)$ & $200(92.2)$ & 0.108 \\
Days to first sit (days) & $52(67.5)$ & $91(41.9)$ & $<0.001$ \\
Standing (n, \%) & $6.0(4.0-10.0)$ & $18(8.3)$ & 0.001 \\
Day to first standing (days) & $8(10.4)$ & $4.0(2.3-6.8)$ & 0.578 \\
Electrical muscle stimulation (n, \%) & $8.5(6.3-15.0)$ & $40(18.4)$ & 0.047 \\
Cycle ergometer training (n, \%) & $2(2.6)$ & $11(5.1)$ & 0.001 \\
\end{tabular}

\section{DISCUSSION}

As a result of placing a dedicated therapist in the ICU and implementing early rehabilitation, the percentage of subjects undergoing rehabilitation intervention was improved significantly, but the improved intervention rate was still only $56.7 \%$. One possible explanation is that patients who stayed in the ICU for up to 2 days were less likely to undergo rehabilitation interventions, which may have suppressed the intervention rate. In phase II, the percentage of reintubations; the length of ICU stay; and the number of days from ICU admission to the day when the patient first underwent reha- bilitation, first underwent sitting, and first underwent standing practice were significantly reduced compared with phase I. However, there were no significant differences between the two groups in the duration of mechanical ventilation, the percentage of extubations and tracheotomies, the length of hospital stay, discharge outcomes, and mortality. Previous reports have suggested that reintubation rates are usually in the range $10-20 \%,{ }^{10)}$ and the main risk factor for reintubation is decreased arterial oxygenation. ${ }^{11)}$ The reason for the decrease in the reintubation rate in phase II may be that early intervention by the dedicated therapist in accordance with the rehabilitation protocol and the active performance of sit- 
Table 3. Comparison of clinical outcomes in patients transferred to another hospital and those discharge to home

\begin{tabular}{lccc}
\hline & $\begin{array}{c}\text { Phase I } \\
\text { Transfer group } \\
(\mathrm{n}=113)\end{array}$ & $\begin{array}{c}\text { Phase II } \\
\text { Transfer group } \\
(\mathrm{n}=134)\end{array}$ & P value \\
\hline SOFA score & $7.5 \pm 3.0$ & $7.8 \pm 2.8$ & 0.438 \\
Rehabilitation intervention (n, \%) & $29(25.4)$ & $90(67.2)$ & $<0.001$ \\
Patients on ventilator (n, \%) & $92(81.4)$ & $118(88.1)$ & 0.077 \\
Extubation (n, \%) & $69(75.0)$ & $84(71.2)$ & 0.324 \\
Duration of ventilation (days) & $5.0(4.0-10.0)$ & $4.0(2.0-8.0)$ & 0.007 \\
Length of ICU stay (days) & $7.0(3.0-14.0)$ & $5.5(3.0-11.0)$ & 0.036 \\
Length of hospital stay (days) & $50.0(28.0-80.3)$ & $53.0(32.8-87.3)$ & 0.232 \\
\hline & Discharge group & Discharge group & P value \\
& $(\mathrm{n}=150)$ & $6.8 \pm 3.4$ & 0.577 \\
SOFA score & $6.5 \pm 2.9$ & $85(50.3)$ & $<0.001$ \\
Rehabilitation intervention (n, \%) & $27(18.0)$ & $112(66.3)$ & 0.065 \\
Patients on ventilator (n, \%) & $112(74.6)$ & $94(83.9)$ & 0.157 \\
Extubation (n, \%) & $100(89.3)$ & $3.5(2.0-7.0)$ & 0.175 \\
Duration of ventilation (days) & $3.0(1.0-6.0)$ & $3.5(2.0-7.0)$ & 0.335 \\
Length of ICU stay (days) & $3.0(2.0-7.0)$ & $29.0(16.0-48.5)$ & 0.375 \\
Length of hospital stay (days) & $31.0(19.8-50.3)$ & & \\
\hline
\end{tabular}

ting and standing increased lower-lung aeration and the tidal volume, which may have contributed to the improvement of arterial oxygenation. However, there were no significant differences between the groups in the duration of mechanical ventilation and the percentages of extubations and tracheotomies. Extubation in patients with less severe illness may not be difficult, but in more severe cases, systemic inflammation and the body fluid balance may have influenced the results. In other words, patients in an unstable general condition had to rely on mechanical ventilation, and the improved lower lung aeration and tidal volume as a result of early intervention alone was not likely to improve outcomes. Several previous studies have shown the effectiveness of early rehabilitation in reducing the length of ICU stay, ${ }^{8,12,13)}$ and this study supports those findings.

The percentage of subjects undergoing rehabilitation intervention increased significantly in phase II compared with phase I. The percentage of rehabilitation patients who performed sitting on the edge of the bed was significantly higher in phase I, but the number of days from ICU admission to the day when the patient first underwent rehabilitation and sitting/standing practice was significantly lower in phase II. These results underline that interventions were performed selectively for patients in a stable general condition in phase I, and therefore the percentage of subjects performing sitting interventions was relatively high. In phase II, early interven- tion was additionally initiated for patients in an unstable general condition who were not targeted in phase I; therefore, the percentage of subjects who performed sitting activities was relatively low in phase II.

There was no significant difference in discharge, transfer, or death outcomes between the two groups. Previous studies and a systematic review have indicated that early rehabilitation has no effect on mortality. ${ }^{14,15)}$ Furthermore, Wright et al. demonstrated that early rehabilitation did not improve physical outcomes at 6 months. ${ }^{16)}$ These results suggest that early intervention does not improve outcomes at discharge and mortality rates. It is likely that the severity of disease, the response to treatment, and the extent of sequelae may affect the outcomes at discharge and mortality rates in patients in a poor general condition who are admitted to the ICU.

Because it is unfeasible to verify the effect of rehabilitation on cases resulting in death, we compared clinical outcomes in subjects discharged to home or transferred to another institution. The discharge group showed no significant difference in any assessment, but in the transfer group, the duration of mechanical ventilation and the length of ICU stay were significantly reduced. In patients with high initial severity scores at the time of ICU admission especially, it has been demonstrated that early exercise rehabilitation may improve functional recovery at discharge. ${ }^{17)}$ Furthermore, stable patients who tend to stay longer in the ICU are more 
likely to benefit from rehabilitation. ${ }^{15)}$ In the current study, the effect of early rehabilitation was likely more pronounced in moderately ill patients who were transferred to another hospital than in fatal cases or in subjects with less severe illness. Rehabilitation in severe cases leading to death is intrinsically ineffective, and less severe cases exhibit a higher reserve capacity for recovery. However, in moderate cases, although the reserve capacity for recovery is maintained, the general condition is unstable, and complications are likely to occur; as a result, the effects of early rehabilitation are likely more easily recognized.

Consequently, there is a certain number of cases in which the effectiveness of rehabilitation can be anticipated, but because severe cases were included in the analysis, the effect may have been weakened in this study. Furthermore, it is necessary to consider possible negative effects of early mobilization. Protein synthesis occurs after the seventh hospital day in survivors of critical illness, ${ }^{18)}$ and the peak concentration of 3-methyl histidine is on the seventh hospital day. ${ }^{19)}$ Therefore, premature early mobilization may increase protein catabolism and risk systemic exhaustion. Nonetheless, some of the myokines released by muscles during exercise have anti-inflammatory effects, ${ }^{20)}$ which is one of the reasons that early mobilization is effective. It is important to set safety standards through rehabilitation protocols to identify such cases and to establish standardized interventions by medical staff who have acquired specific skills and experience.

There were some limitations to the present study. First, the influence of gender cannot be excluded. Second, it was a single-center retrospective study. Prospective multicenter studies are required to obtain more robust results. Third, previous reports have suggested that early rehabilitation in the ICU can reduce the incidence of delirium, ${ }^{21)}$ but delirium could not be assessed in the current study. The presence or absence of delirium may be related to the duration of mechanical ventilation, extubation, and the length of ICU stay. Fourth, although there were no major differences in the severity of illness nor in the extent of ventilator usage between the two groups, the diseases of the subjects were diverse. This fact may have influenced outcomes because of differences in the courses of specific diseases. In the future, it will be necessary to perform additional studies in which the subjects have the same category of disease.

\section{CONCLUSION}

In this single-center retrospective study, the rate of early intervention in the ICU increased after the creation of a rehabilitation protocol and the assignment of a dedicated therapist. As a result, the percentage of subjects undergoing reintubation; the length of ICU stay; and the number of days between ICU admission and the first rehabilitation, the first sitting exercise, and the first standing exercise decreased significantly. Moreover, the duration of mechanical ventilation and the length of ICU stay were significantly reduced in the transfer group. Prospective multicenter studies are required to further explore these findings.

\section{CONFLICTS OF INTEREST}

None of the authors have any conflicts of interest to disclose.

\section{REFERENCE}

1. Drakulovic MB, Torres A, Bauer TT, Nicolas JM, Nogué S, Ferrer M: Supine body position as a risk factor for nosocomial pneumonia in mechanically ventilated patients: a randomised trial. Lancet 1999;354:1851-1858. DOI:10.1016/S0140-6736(98)12251-1, PMID:10584721

2. Devlin JW, Skrobik Y, Gélinas C, Needham DM, Slooter AJ, Pandharipande PP, Watson PL, Weinhouse GL, Nunnally ME, Rochwerg B, Balas MC, van den Boogaard M, Bosma KJ, Brummel NE, Chanques G, Denehy L, Drouot X, Fraser GL, Harris JE, Joffe AM, Kho ME, Kress JP, Lanphere JA, McKinley S, Neufeld KJ, Pisani MA, Payen JF, Pun BT, Puntillo KA, Riker RR, Robinson BR, Shehabi Y, Szumita PM, Winkelman C, Centofanti JE, Price C, Nikayin S, Misak CJ, Flood PD, Kiedrowski K, Alhazzani W: Executive summary: clinical practice guidelines for the prevention and management of pain, agitation/sedation, delirium, immobility, and sleep disruption in adult patients in the ICU. Crit Care Med 2018;46:1532-1548. DOI:10.1097/ CCM.0000000000003259, PMID:30113371

3. Kress JP, Pohlman AS, O’Connor MF, Hall JB: Daily interruption of sedative infusions in critically ill patients undergoing mechanical ventilation. N Engl J Med 2000;342:1471-1477. DOI:10.1056/ NEJM200005183422002, PMID:10816184

4. Pang Y, Li H, Zhao L, Zhang C: An established early rehabilitation therapy demonstrating higher efficacy and safety for care of intensive care unit patients. Med Sci Monit 2019;25:7052-7058. DOI:10.12659/ MSM.916210, PMID:31537777 
5. Schefold JC, Bierbrauer J, Weber-Carstens S: Intensive care unit-acquired weakness (ICUAW) and muscle wasting in critically ill patients with severe sepsis and septic shock. J Cachexia Sarcopenia Muscle 2010;1:147-157. DOI:10.1007/s13539-010-0010-6, PMID:21475702

6. Norrenberg M, Vincent JL European Society of Intensive Care Medicine: A profile of European intensive care unit physiotherapists. Intensive Care Med 2000;26:988-994. DOI:10.1007/s001340051292, PMID:10990117

7. Taito S, Sanui M, Yasuda H, Shime N, Lefor AK, Japanese Society of Education for Physicians and Trainees in Intensive Care (JSEPTIC) Clinical Trial Group: Current rehabilitation practices in intensive care units: a preliminary survey by the Japanese Society of Education for Physicians and Trainees in Intensive Care (JSEPTIC) Clinical Trial Group. J Intensive Care 2016;4:66. DOI:10.1186/s40560-016-0190-z, PMID:27800164

8. Matsuki R, Kojima N, Watanabe K, Hotta A, Kubori Y, Oura K, Morisawa T, Koyama H, Ebisu T, Hashino $\mathrm{T}$ : Impact of a rehabilitation protocol and a dedicated therapist in the intensive care unit on physical function and activities of daily living. Prog Rehabil Med 2020. doi: DOI:10.2490/prm.20200027, PMID:33163685

9. Boles JM, Bion J, Connors A, Herridge M, Marsh B, Melot C, Pearl R, Silverman H, Stanchina M, Vieillard-Baron A, Welte T: Weaning from mechanical ventilation. Eur Respir J 2007;29:1033-1056. DOI:10.1183/09031936.00010206, PMID:17470624

10. Thille AW, Richard JC, Brochard L: The decision to extubate in the intensive care unit. Am J Respir Crit Care Med 2013;187:1294-1302. DOI:10.1164/rccm.2012081523CI, PMID:23641924

11. Fujii E, Fujino K, Tanaka-Mizuno S, Eguchi Y: Variation of risk factors for cause-specific reintubation: a preliminary study. Can Respir J 2018;2018:1-6. DOI:10.1155/2018/3654251, PMID:30510604

12. Dong Z, Yu B, Sun Y, Fang W, Li L: Effects of early rehabilitation therapy on patients with mechanical ventilation. World J Emerg Med 2014;5:48-52. DOI:10.5847/ wjem.j.issn.1920-8642.2014.01.008, PMID:25215147

13. Yosef-Brauner O, Adi N, Ben Shahar T, Yehezkel E, Carmeli E: Effect of physical therapy on muscle strength, respiratory muscles and functional parameters in patients with intensive care unit-acquired weakness. Clin Respir J 2015;9:1-6. DOI:10.1111/crj.12091, PMID:24345055
14. Tipping CJ, Harrold M, Holland A, Romero L, Nisbet T, Hodgson CL: The effects of active mobilisation and rehabilitation in ICU on mortality and function: a systematic review. Intensive Care Med 2017;43:171-183. DOI:10.1007/s00134-016-4612-0, PMID:27864615

15. Waldauf P, Jiroutková K, Krajčová A, Puthucheary Z, Duška F: Effects of rehabilitation interventions on clinical outcomes in critically ill patients: systematic review and meta-analysis of randomized controlled trials. Crit Care Med 2020;48:1055-1065. PMID:32345834

16. Wright SE, Thomas K, Watson G, Baker C, Bryant A, Chadwick TJ, Shen J, Wood R, Wilkinson J, Mansfield L, Stafford V, Wade C, Furneval J, Henderson A, Hugill K, Howard P, Roy A, Bonner S, Baudouin S: Intensive versus standard physical rehabilitation therapy in the critically ill (EPICC): a multicentre, parallel-group, randomised controlled trial. Thorax 2018;73:213-221. DOI:10.1136/thoraxjnl-2016-209858, PMID:28780504

17. Ahn JY, Song JE, Ann HW, Jeon Y, Ahn MY, Jung IY, Kim MH, Jeong W, Jeong SJ, Ku NS, Kim JM, $\mathrm{Na}$ S, Cho SR, Choi JY: Effects of early exercise rehabilitation on functional recovery in patients with severe sepsis. Yonsei Med J 2018;59:843-851. DOI:10.3349/ ymj.2018.59.7.843, PMID:30091317

18. Puthucheary ZA, Rawal J, McPhail M, Connolly B, Ratnayake G, Chan P, Hopkinson NS, Padhke R, Dew T, Sidhu PS, Velloso C, Seymour J, Agley CC, Selby A, Limb M, Edwards LM, Smith K, Rowlerson A, Rennie MJ, Moxham J, Harridge SD, Hart N, Montgomery HE: Acute skeletal muscle wasting in critical illness. JAMA 2013;310:1591-1600. DOI:10.1001/jama.2013.278481, PMID:24108501

19. Wandrag L, Brett SJ, Frost GS, Bountziouka V, Hickson M: Exploration of muscle loss and metabolic state during prolonged critical illness: implications for intervention? PLoS One 2019;14:e0224565. DOI:10.1371/ journal.pone.0224565, PMID:31725748

20. Pedersen BK, Febbraio MA: Muscle as an endocrine organ: focus on muscle-derived interleukin-6. Physiol Rev 2008;88:1379-1406. DOI:10.1152/physrev.90100.2007, PMID:18923185

21. Schweickert WD, Pohlman MC, Pohlman AS, Nigos C, Pawlik AJ, Esbrook CL, Spears L, Miller M, Franczyk M, Deprizio D, Schmidt GA, Bowman A, Barr R, McCallister KE, Hall JB, Kress JP: Early physical and occupational therapy in mechanically ventilated, critically ill patients: a randomised controlled trial. Lancet 2009;373:1874-1882. DOI:10.1016/S01406736(09)60658-9, PMID:19446324 